\title{
Effects of Training with Impairment Based Therapeutic Intervention Program for Improvement in Function and Independence in an Individual Diagnosed with Transverse Myelitis
}

Leah Jamison DPT ${ }^{1}$, Mallory Spoonts PT, DPT, NCS ${ }^{2}$, Martín G. Rosario PT, PhD, ATRIC, CSFI ${ }^{1^{*}}$

${ }^{1}$ Physical Therapy Program, Texas Woman's University, Dallas Campus; Texas; United States.

${ }^{2}$ Baylor Scott and White Institute for Rehabilitation, Grapevine Texas, United States.

\author{
Article Details \\ Article Type: Case Report \\ Received date: $09^{\text {th }}$ May, 2020 \\ Accepted date: $22^{\text {th }}$ June, 2020 \\ Published date: $24^{\text {th }}$ June, 2020
}

"Corresponding Author: Martín G. Rosario PT, PhD, ATRIC, CSF, Assistant Professor, School of Physical Therapy, Texas Woman's University, 5500 Southwestern Medical Ave, Dallas Campus; Texas, United States. E-mail: mrosario1@twu.edu Citation: Jamison, L., Spoonts, M., \& Rosario, M.G. (2020). Effects of training with impairment based therapeutic intervention program for improvement in function and independence in an individual diagnosed with Transverse Myelitis. J Rehab Pract Res 1(1):106. https://doi.org/10.33790/jrpr1100106

Copyright: $(2020$, This is an open-access article distributed under the terms of the Creative Commons Attribution License 4.0, which permits unrestricted use, distribution, and reproduction in any medium, provided the original author and source are credited.

\begin{abstract}
s
Background and Purpose: Transverse Myelitis (TM) is a unique illness differentiated by inflammation of the spinal cord. Various characteristics of TM are related, but not limited to, infections, immune system disorders, and other disruptions that distort myelin. Heretofore, this disorder has numerous clinical manifestations; the research on how to physically rehabilitate and promote the quality of life in individuals withstanding from this illness is limited. The goal from this case report is to arise with a promising treatment plan for an individual with TM to restore as much function and independence as possible.
\end{abstract}

Case Description: The emphasis of this work is to report the aftermath and rehabilitation approach of a 44-year-old male diagnosed with TM. He was diagnosed with TM on April 3, 2019, at the Emergency Department after a sudden onset of chest pain. The patient displayed paresthesia from the bilateral nipple line (T4) to the bilateral lower extremities (BLE) (S1). The right lower extremity (RLE) unchanged hot and cold sensation while the left lower extremity (LLE) displays unscathed deep pressure and pain sensation. Similarly, he exhibits muscle weakness (RLE $>$ LLE), gait deviations (right knee buckling), fatigue, and risk for falls based on the functional tests (6MWT, TUG, and FTSTS) chosen for this case study. The patient's expectation involved ambulating independently with the least restrictive device, along with other physical therapy objectives related to his goal.

Interventions: Treatment program was developed based on the patient's impairment rather than the diagnosis. Patient education, balance (core activation), gait activities, strength (pilate based), and eccentric exercises were some of these patients' interventions.

Outcomes: The patient's frequency and duration varied due to insurance complications; nevertheless, the set duration was prescribed two to three times a week for two months. Based on retesting the selected functional tests, he was able to ambulate with a least restrictive device and perform a decrease in right knee buckling during mid-stance.

Discussion/Conclusion: In this case study, the patient was unable to reach his personal goal of walking independently. Withal, he did fulfill most set physical therapy goals and exhibit advancement in functional acti-vities inside and outside the clinic. With the patients' rare diagnosis, minimal literature was available for the related interventions for these specific symptoms. Future studies attending similar cases should focus on examining long term benefits of physical therapy as well as a more extended period of intervention to obtain a clear clinical picture of the advantages of such treatment.

\section{Background}

Transverse Myelitis (TM) is an unusual disorder distinguished by inflammation of the spinal cord [1,2]. Factors of TM comprise infections, immune system disorders, and other disturbances that may damage or destroy myelin 11 . The specific etiology of $60 \%$ of TM cases may be unknown despite the existence of inflammatory mechanisms [1, 3]. However, the remaining $40 \%$ is associated with conditions such as; immune system disorders, viral or bacterial infections, inflammatory disorders (including Lupus, and scleroderma), and vascular disorders [1,3]. TM is an ailment that strikes all age groups despite family history, gender, or race [1]. The spinal cord carries motor nerve fibers to the limbs and trunk as well as sensory strands from the body back to the brain [2]. Inflammation within the spinal cord hinders these pathways and causes uncommon presenting symptoms [2]. TM commonly expresses with muscle weakness and paralysis, starting up with the legs and arms, depending on the level the cord is being affected [2]. Sensory input, such as light touch, pain, and temperature, are generally diminished, along with a tight banding sensation around the trunk [2]. Other indications that are common in TM patients are sexual dysfunction, increased bladder and bowel incontinence, spasticity, fatigue, and depression [2]. Literature reports the recovery patterns with this type of diagnosis showing an even distribution between little to minor symptoms, moderate degrees of permanent disability, and no recovery with severe functional disability [2].

Rehabilitation after any type of spinal cord injury improves functional outcomes; however, TM has other factors that can negatively impact rehabilitation participation. There is a lack of research detailing physical therapy (PT) interventions for patients with TM. This case study highlights the process of diagnosis, treatment, and outcomes of TM in a 44 year old male who has integrated an alternative lifestyle with his family secondary to his condition.

\section{Case Presentation}

History

The patient is a forty-four $y / 0$ male, married with four children. 
Past medical history consists of hyperlipidemia and osteoarthritis in bilateral hips. He currently works at home. He was playing soccer five times a week, however, reduced to once a week due to right hip pain for the past four years. In April 2019, the patient was on his way to work when withstanding an abrupt onset of chest pain. After arriving at work five minutes later, he started to experience numbness and tingling in the right toes. While he endured these symptoms, one of his co-workers drove him to the emergency room (ED). Thereupon arriving at the ED, where he was diagnosed, the right leg became paralyzed along with his left leg at twenty minutes. After being released from the hospital, he was assigned to an inpatient rehabilitation facility for treatment (two to three times a week for six to eight weeks). At the inpatient rehab facility, treatment focused on ambulating with a rolling walker (RWW), knee brace, and posterior leaf, all for the right lower extremity (RLE). After inpatient rehab, he was independent with transfers. Due to leg fatigue, he could only stand for 30 minutes with a rolling walker. The patient got a Neuromuscular Machine Electrical Stimulation (NMES) to assist with quad activation at home. In December 2019, the patient was advised about surgery for a total hip replacement due to osteoarthritis. Then, the patient completed a week of inpatient rehab again for post right total hip replacement. Currently, for ambulation, he uses a manual wheelchair for the community and the rolling walker for the household. When prolonged lying supine or sitting, the right lower extremity tends to draw up in hip flexion involuntarily, causing pain and tension in the lower extremities. He is currently prescribed Gabapentin and Baclofen to aid in decreasing nerve pain and spasticity. The patient's goal is to ambulate independently without an assistant device.

\section{Systems Review}

A minimal systems review revealed the following; first, the integumentary system had some scarring on the upper right thigh from an anterior approach right total hip replacement. Moreover, there were three minor circular burn marks on the right mid-thigh due to high voltage NMES during the inpatient rehab period. The patient exhibited a standard height with overweight body composition. The cardiopulmonary system seemed to be within functional limitations. He was oriented to person, place, and time with appropriate communication. The neurologic system displays paresthesia from the bilateral nipple line (T4) to bilateral lower extremities (S1). The right lower extremity unchanged hot and cold sensation, while the left lower extremity (LLE) displays unscathed deep pressure and pain sensation.

\section{Examination}

An objective examination was conducted to determine baseline data, such as muscular strength, reflexes, gait, and functional tests. Table 1 highlights the results of the musculoskeletal examination. Our case report exhibited weakness in addition to decreased muscle activation and strength in the RLE than in the LLE. Altogether, the strength on the RLE averages out to $-2 / 5$ on other muscle activations (knee extension, knee flexion, and ankle plantarflexion). Ankle clonus was tested with the patient placed in the supine position, for reflex integrity testing (upper motor neuron test) with the additional observation of multiple beats bilaterally. A score of two was observed on The Modified Ashworth Scale (increase tone; affect easily) for right hamstrings and 1 (slight increase; minimal resist at end ROM) for right quadriceps. Functional tests (Table 2) were used as an indication of improvement for functional outcomes. The five-time sit to stand (FTST) was used for indication of strength/power and risk for falls. The time for FTSTS 26.17 seconds with right upper

\begin{tabular}{|l|l|l|}
\hline MMT of LE & Left & Right \\
\hline Hip flexion & $3 / 5$ & $+1 / 5$ \\
\hline Knee Extension & $4 / 5$ & $-2 / 5$ \\
\hline Knee Flexion & $4 / 5$ & $-2 / 5$ \\
\hline Ankle Dorsiflexion & $4 / 5$ & $-1 / 5$ \\
\hline Ankle Plantar Flexion & $-3 / 5$ & $-2 / 5$ \\
\hline
\end{tabular}

Table 1.- Initial Results of MMT

\begin{tabular}{|l|l|l|}
\hline Functional Test & Patients Value & Norm Value \\
\hline FTSTS & 26.17 second & $\begin{array}{l}>14.8 \text { seconds indicate low strength/ } \\
\text { power and risk for falls [4]. }\end{array}$ \\
\hline TUG & 45.73 second & $\begin{array}{l}>15 \text { seconds indicate risk for falls } \\
{[5] .}\end{array}$ \\
\hline 6MWT & 200 feet & 1050 feet for men [6]. \\
\hline
\end{tabular}

extremity on the RWW and left upper extremity pushing from the mat table at 19-inch height [4]. Timed up and go (TUG) used for indication of the risk of falls, and this case report was noted at $45.73 \mathrm{sec}$ with RWW [5]. The six-minute walk test (6MWT) was performed to show endurance and walking capacity improvements; in the current case report, 6MWT was recorded as 200 feet [6]. Ambulation was completed with observation, which showed fair gait speed and functional safety with negotiation. The patient ambulated with an RWW, right posterior leaf orthosis, and right knee brace locked into extension to prevent knee flexion (knee buckling) and assist with weight-bearing. A compensation pattern was identified while walking; the patient exhibited some right hip hiking and circumduction to advance the right lower extremity. Additionally, insufficient right dorsiflexion and hip flexion, which led to vaulting on the left lower extremity during stance phase to assist in right toe clearance. Finally, while walking without a brace, a flat foot strike or landing on the right toe in addition to right knee hyperextension during bearing weight was noted.

\section{Evaluation}

Due to the rarity of TM, the full clinical manifestation of this diagnosis is unknown. Symptoms presentation includes pain, loss of sensation, urinary retention, and weakness [7]. Upon initial examination and evaluation, the patient presented impaired balance, difficulty walking, and muscular weakness secondary to the diagnosis of TM. Prior medical management included gait training in the parallel bars with three-person assistance, standing frame, and toe taps on stairs with three people assistance [Figure 1.1-1.3]. When testing the patient's strength, there was significant weakness on the right lower extremity and some in the left lower extremity. At the 


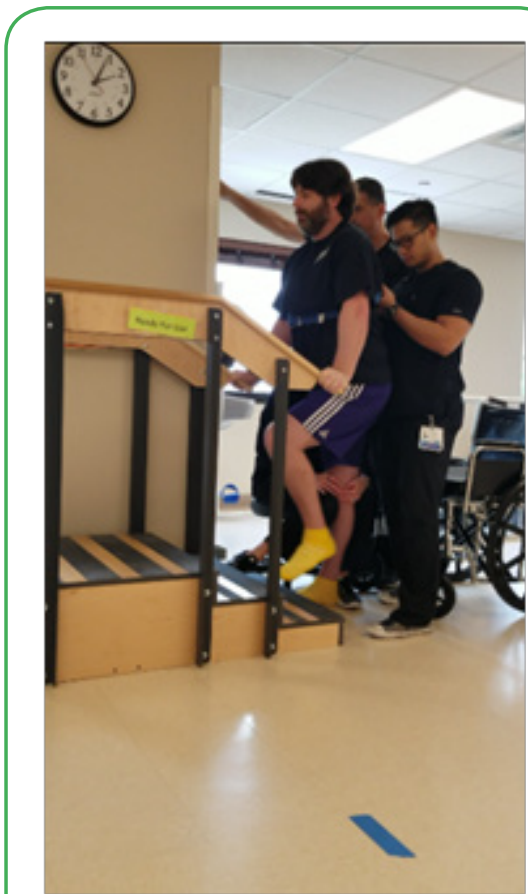

Figure 1.1: Toe taps on stairs with three-person assistance at IP Rehab.

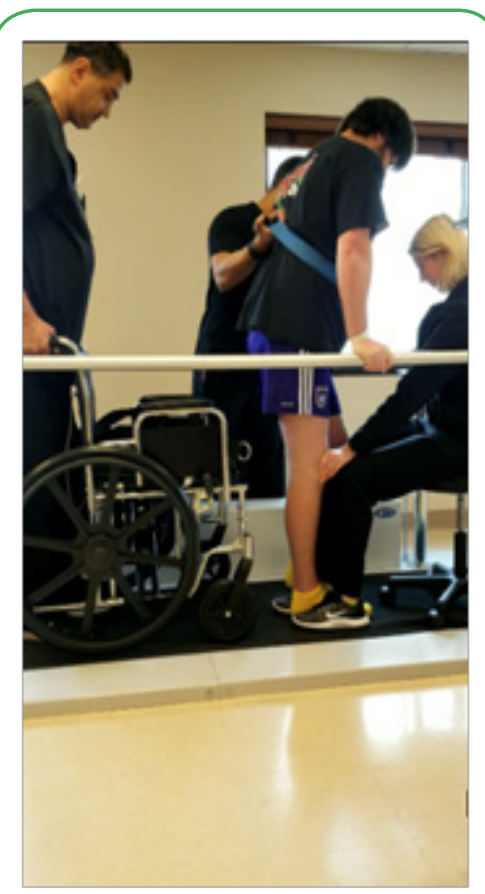

Figure 1.2: Gait training in parallel bars with three-person assistance at IP Rehab. time of his initial evaluation, the patient could perform right knee extension, which he stated was unable to accomplish. When performing gait assessments, the patient ambulated with a forward rolling walker, right posterior leaf orthosis, and right knee brace to assist with maintaining knee extension due to his muscular weakness. A potential barrier to rehab the patient exhibits is being limited in the amount of physical activity due to chronic right hip pain.

For this case report, the International Classification of Functioning, Disability, and Health (ICF) was used to provide a descriptive profile

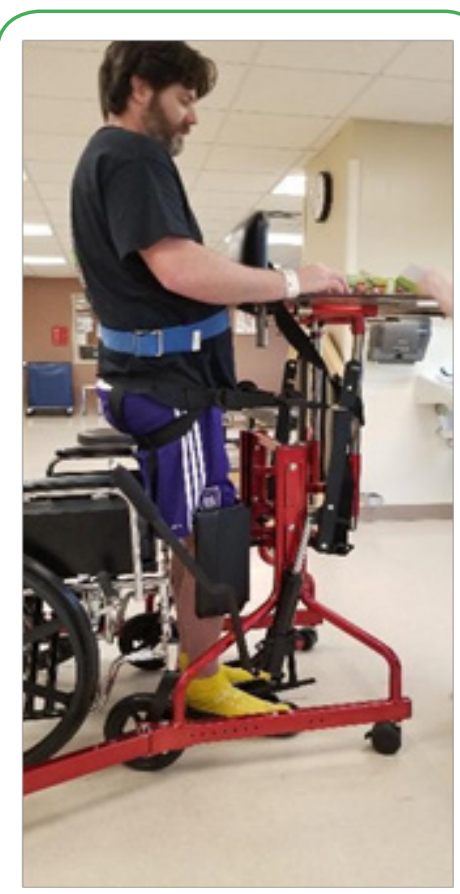

Figure 1.3: Standing frame at IP Rehab. of the individual's pattern of functioning, however, not a general answer about whether he or she is disabled [8]. In clinical settings, the ICF model can be used in its full range as a framework for rehabilitation programming [9]. Based on this literature, an ICF model [Table 3] was recorded from the initial evaluation. With this model in mind, our physical therapy focus was to improve gait mobility and increase strength to achieve not only his goal to walk independently but to be successful in his other functional activities.

\section{ICF Model}

Medical Diagnosis: Acute Transverse Myelitis in demyelinating disease of CNS (G37.3)

Physical Therapy Diagnosis: Other Abnormalities of Gait and Mobility (R26.89); Unsteadiness on Feet (R26.81); Weakness (R53.1).

\begin{tabular}{|c|c|c|}
\hline Body Functions \& Structures & Activity Limitations & Participation Restrictions \\
\hline $\begin{array}{l}\text { 1. Pain } \\
\text { 2. Abnormal Gait Characteristics: } \\
\text { - Compensation with hip hiking } \\
\text { and circumduction to advance } \\
\text { RLE. } \\
\text { - No hip flexion, knee flexion, } \\
\text { and dorsiflexion on RLE. } \\
\text { - Presence of vaulting on the LLE } \\
\text { to assist with right toe clearance. } \\
\text { 3. Weakness secondary to } \\
\text { diagnosis } \\
\text { 4. Decreased muscular endurance } \\
\text { 5. Marked increase tone that } \\
\text { is affected easily in bilateral } \\
\text { hamstrings. } \\
\text { 6. Hyperreflexia } \\
\text { 7. Right Total Hip Arthroplasty } \\
\text { 8. Impaired motor control in BLE }\end{array}$ & $\begin{array}{ll}- & \text { Running } \\
- & \text { Jumping } \\
- & \text { Walking independently } \\
- & \text { Prolonged Standing } \\
- & \text { Prolong sitting } \\
- & \text { Ascending stairs. }\end{array}$ & $\begin{array}{ll}\text { - } & \text { Inability to play soccer } \\
\text { - } & \text { Inability to participate with } \\
\text { extra activities with his kids. } \\
\text { - } & \text { Driving }\end{array}$ \\
\hline
\end{tabular}

Table. 3 to be Cont........ 
9. Diminished sensation from abdominal area (T4) to BLE (S1).

RLE: hot and cold sensation

LLE: deep pressure sensation

10. Impaired balance stability

\begin{tabular}{|c|c|}
\hline \multicolumn{2}{|r|}{ Contextual Factors } \\
\hline $\begin{array}{l}\text { Environmental Factors } \\
\text { Negatives: } \\
\text { 1. Not driving } \\
\text { 2. Two story home } \\
\text { Positives: } \\
\text { 1. Work from home } \\
\text { 2. Occasionally goes to Neuro fit } \\
\text { gym } \\
\text { 3. Wheelchair, walker, and crutch } \\
\text { accessibility around the house. }\end{array}$ & $\begin{array}{l}\text { Negatives: } \\
\text { 1. Post-op Right THR } \\
\text { 2. Possible Left THR in the future } \\
\text { 3. Comorbidities: OA, and high cholesterol. } \\
\text { Positives: } \\
\text { 1. 44-year-old male } \\
\text { 2. Highly Motivated } \\
\text { 3. Good understanding of diagnosis and prognosis } \\
\text { 4. Great family support system } \\
\text { 5. Compliant with exercise } \\
\text { 6. NMES at home from IP Rehab for quadricep and tibialis anterior muscle } \\
\text { 7. Previous Physical Therapy Treatment }\end{array}$ \\
\hline
\end{tabular}

A Re-Evaluation was done in February 2020, where his presentation dramatically improved from the initial onset in December 2019. The patient presented with an increase in endurance and strength. During the TUG, the patient showed improvements in gait, completing the task in half the time ( 23 seconds) from the initial evaluation (45.73 seconds). Also, a second trial of TUG was performed with forearm crutches with supervision in 26 seconds with the goal of improvement in community ambulation and cadence. The patient demonstrated an increase in endurance by accomplishing $450 \mathrm{ft}$ without rest on the 6MWT compared to his initial testing in which he performed 200 feet with RW. When observing his gait, there was a decrease in left lower extremity vaulting for right foot clearance and an increase in the right knee and hip flexion for toe clearance. The patient can perform ambulation without assistive devices (posterior leaf orthosis or knee brace); however, his right knee buckles into hyperextension, possibly due to right gastrocnemius weakness, gastrocnemius tightness, or quadriceps weakness. The patient now performs household and community ambulation with forearm crutches instead of RW; despite, he still has a manual wheelchair close by in case of fatigue. Other functional testing's were deferred to the next reassessment due to timing constraints.

\section{Diagnosis}

The patient was diagnosed in early April 2019 after experiencing nonspecific chest pain on his way to work. A magnetic resonance image (MRI) was obtained on 4/3/19 that revealed a complete resolution of cord edema at the T2 spinal level [Figure 2], along with multiple blood tests and lumbar puncture. Also, the patient was examined and tested for Guillain Barre syndrome and Lupus before the final medical diagnosis. From the differential testing, the referring physician made a medical diagnosis of Acute Transverse Myelitis, a demyelinating disease of the central nervous system (G37.3). After the initial evaluation, physical therapy diagnoses were made secondary to the primary medical diagnosis and recent right total hip replacement to describe functional deficits. The following are the physical therapy diagnosis: Other abnormalities of gait and mobility (R26.89), Presence of artificial right hip joint (Z96.641), Unsteadiness on feet (R26.81), and Weakness (R53.1). The deficits listed above were used to create functional short term and long-term goals.

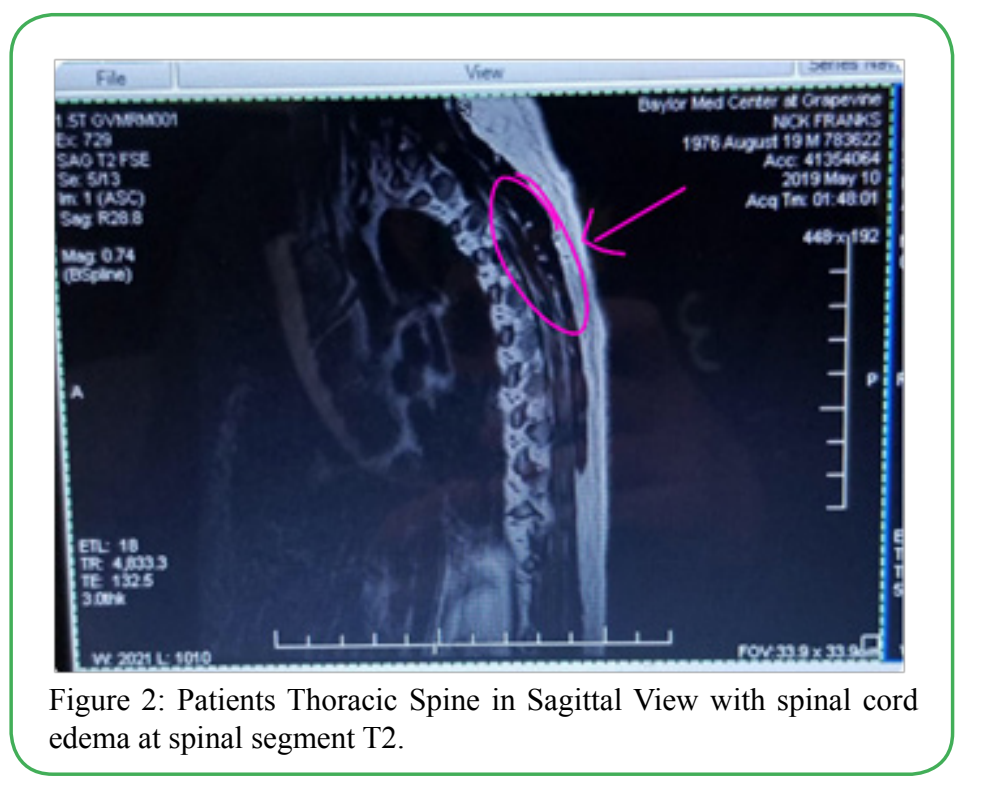




\section{Prognosis and Plan of Care}

Most people that are diagnosed with transverse myelitis have a partial recovery, with the peak of the improvement befalling within the first three months after initial onset [10]. Because the patient commenced IP Rehab one week after being diagnosed, his prognosis was good to excellent, leading to an increase in his functional outcomes. Withal, some individuals are left with moderate disabilities, such as trouble walking and nerve sensitivity. In contrast, other subjects exhibit more permanent effects in addition to other complications resulting in a poor recovery [10]. Due to the aforesaid, the prognosis is inconsistent and unpredictable, resulting in either a full recovery or culminating with permanent sequelae of impairments [10]. With this evidence in reason, this patient's prognosis for rehabilitation should be poor. Although, with insufficient evidence, it is difficult to state the precise prognosis for someone diagnosed with acute TM. The highest determining factor for an individual patient's physical therapy encounter does play a large role, which was determined to be good.

The patient's primary goal was to walk independently with no assistive device for community ambulation and to return to normal function. People diagnosed with acute TM will have trouble walking as a permanent sequelae [10], however, the patient's presentation and compliance make this a plausible goal. Although that was the patient's primary goal, physical therapy goals were made as well. Each goal was listed under the patients' main problems and linked to subjective and objective examinations. Several clinical manifestations were identified. First, a diminished time during prolonged standing and walking; the short-term goal (STG) was that the patient would report tolerating walking/standing for 30 minutes to assist with household ambulation. The long-term goal (LTG) was that the patient would tolerate walking/standing for one hour to be engaging during household and community ambulation activities. The second complication was associated with the 6-minute walk test (6MWT) functional analysis. The associated LTG for the 6MWT was based on the patient's capability to walk 600 feet with the least restrictive device to assist with household and community ambulation. Issue three was associated with the functional test Timed up and go (TUG); the STG was accomplishing the TUG in 20 seconds or less with a least restrictive device, and the LTG was performing the TUG in 15 seconds or less with the least restrictive device to decrease the risk of falling. Difficulty four consisted of the manual muscle testing (MMT) of the bilateral lower extremity (BLE); the STG was a gross appraisal of 3 out of 5, and the LTG was a gross assessment of 4- out of 5 to assist with gait. All previously mentioned, STG had a time frame of 2 weeks, while the LTG had a time frame of 4 weeks. Despite, as expected, with a medical complexity such as the current case report, medical diagnosis, and uncertain prognosis, some goals were extended based on the plan of care. Accordingly, we recommended that the patient attends rehabilitative therapy for two visits a week with an expected duration of two months.

\section{Interventions}

Therapeutic exercises were ascertained focused on the physical therapists' and patient's goals for improvement in motor control, balance, flexibility, and patient education. After the initial evaluation, the first mode of treatment used was NMES, patient education for muscular re-education and strengthening within the home exercises program. NMES is used frequently in physical therapy to increase voluntary strength in partial muscles in people with spinal cord injury (SCI) or similar presentation. In this report, NMES was used to increase strength and re-education in bilateral quadriceps to promote an increase in motor control while performing gait activities. Following this, the next item in the treatment plan was motor control exercises while in supine and quadruped positions, including heel slides, short arc quad (SAQ), and knee extension full arc. Due to muscle weakness, the patient required active assistance for hip and knee control on the RLE due to an increase in tone and weak hip extensor muscles. Motor control evolves from a complex set of processes that govern posture and movement [11]. Through literature, we implemented a selection of motor control exercises proven to be effective in restoring efficient muscle use and gaining optimal control to improve gait training in hemiparetic stroke patients [12]. The previously mentioned type of intervention was selected based on the patients' similar presentation of decreased muscle control and the deficit of extensor muscle use in the RLE, as seen in a stroke patient. The patient also participated in balance activities like standing weight shifting for improvement in weight distribution and postural control while performing functional tasks. A selection of core activation activities (until motor fatigue) for the increase in balance stability, included modified plank, ball rollouts in a tall kneeling position, and quadruped alternate leg extension. Too, we implemented flexibility exercises in the plan of care towards the end of each treatment session, such as passive hamstring stretching for 3 sets with 30- second holds; for a decrease in muscle tightness and prevention of loss of full range of motion due to increase in tone. Other flexibility exercises included prone on elbows to cobra for a decrease in tension in the abdominal area with resting breaks between each exercise throughout treatment, as shown in Figure 5.

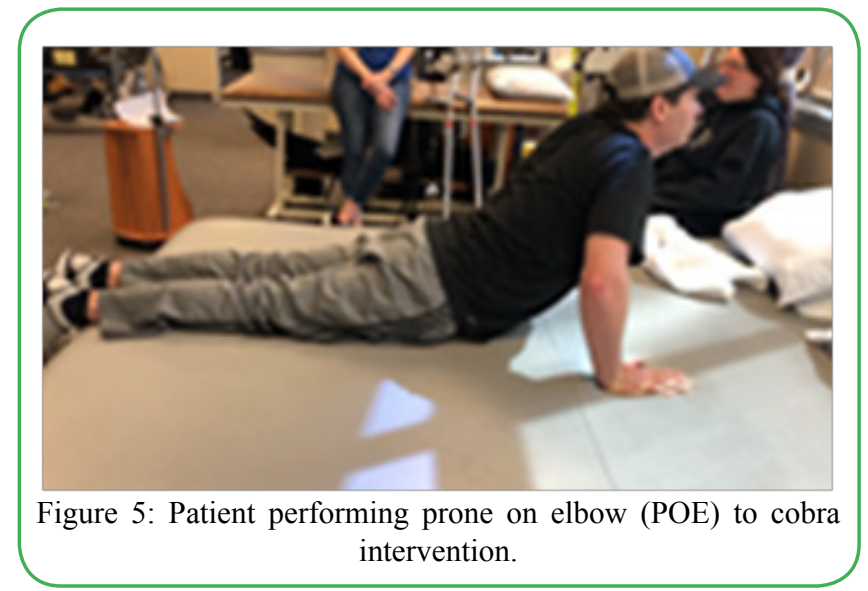

For the patient's safety, strength training activities were performed in the parallel bars, supine position, and tall kneeling on the edge of the mat at stand by assistance. Strength exercises consisted of an increase in quadriceps and hamstring muscle endurance and eccentric activities for different muscle groups. Initial quadriceps and hamstring strengthening exercises included leg press machine bilaterally and unilaterally and step- ups in the parallel bars. The leg press machine was actively assisted by a physical therapist (PT)and set to low intensity and repetitions, additionally on the RLE due to an increase in extensor tone and insufficient knee flexion motor control. Throughout the patients' frequency in and duration in the clinic, the exercises progressed to an increase in repetitions, intensity, and no assistance in RLE control for improvement in functional tasks. Stepups were attained on a 2 -inch step in the parallel bars with minimal to no 
upper extremity assistance for the decrease in trunk momentum and an increase in hip flexion. Frequent touches on parallel bars were made after a complete ascending reciprocal cycle of RLE on the step for balance stability due to excessive right knee hyperextension when weight-bearing. A progression to a 6-inch step was promoted for an improvement in right hip flexion initiation of the iliopsoas muscle. Eccentric exercise activities were selected to assist in challenging the muscles, which, according to literature, can lead to stronger muscles and a boost in metabolic rate. Literature states that eccentric actions generate greater force than isometric and concentric contractions [13].

Moreover, eccentric contractions are functional, occur in everyday

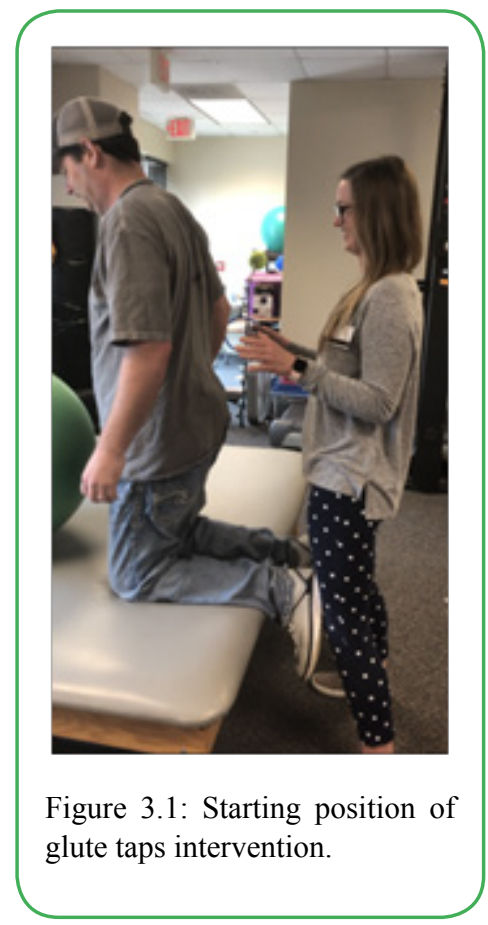

Next, static heel raises were initially utilized for the functional strengthening of the gastrocnemius and soleus muscles, specifically the RLE over the LLE. The gastrocnemius muscle produces knee flexion during loading response, mid stance, and single-leg stance phase in the gait cycle, which all require full weight bearing on a single limb, thus necessary to work on this case report [15]. Due to the decrease in right calf strength, the patient was unable to perform static heel raises without overly compensating on the left lower

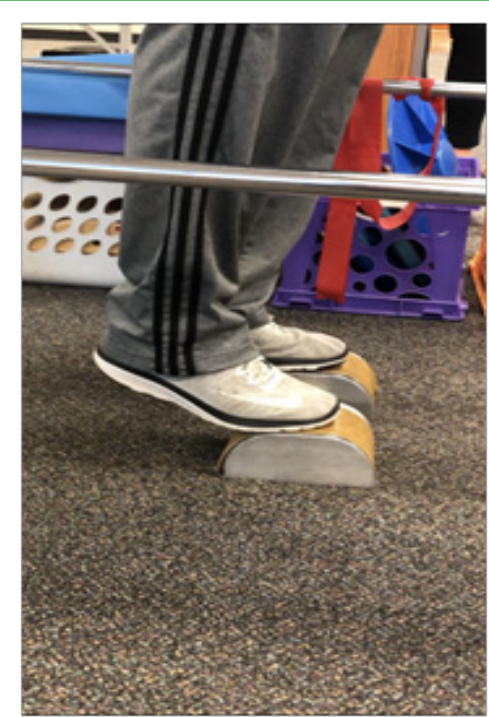

Figure 4.1: Eccentric calf exercise with LLE ascending heel raises for RLE heel raise assistance and concentric contraction of LLE. motor activities, such as walking and standing and enable the dissipation of mechanical energy during body deceleration, like descending stairs, and facilitates the conversion of kinetic energy into elastic energy of tendons [13]. Pilates like exercises was selected for enhancing muscle mass, specifically in the hamstring muscles. Pilates involves low and intermediate intensity resistance and repetition that match but also improve the patient's physical ability [14]. One utilized Pilates technique was Glute Taps in a tall kneeling position on the edge of the mat to increase strength in the right hamstring. Initially, the patient required active assistance by PT for the prevention of extensor tone on RLE. This intervention was performed until motor fatigue or recognition of compensation on the LLE. [Figure 3.1-3.2].

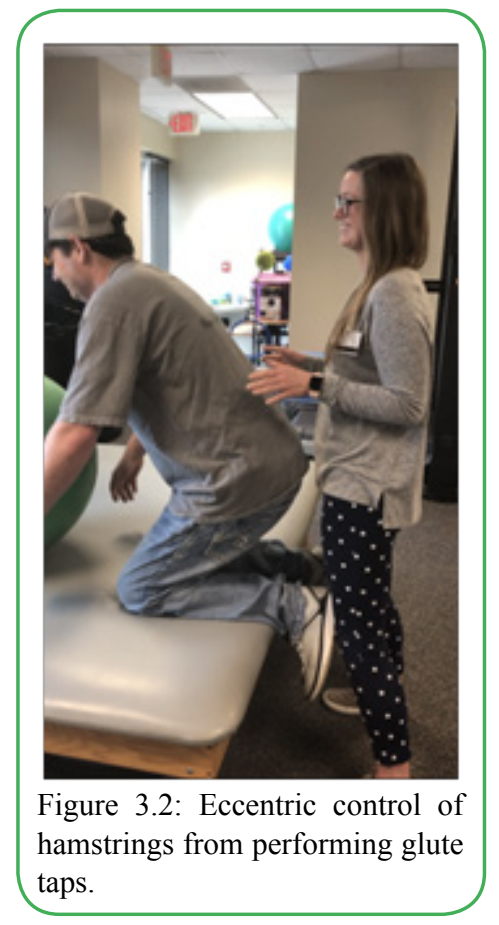

extremity, thus minimizing the purpose of right calf muscle activity. Eccentric calf raises were utilized as a modification for an increase in right calf strength. The subject would execute a heel raise with the LLE for the concentric contraction, then slowly descend the right heel down for an eccentric control of the RLE. All those above were achieved on a slant board for a boost in assistance. [Figure 4.1-4.2]. Our goal from this intervention is to help strengthen these muscles to decrease right knee hyperextension when mid-stance.

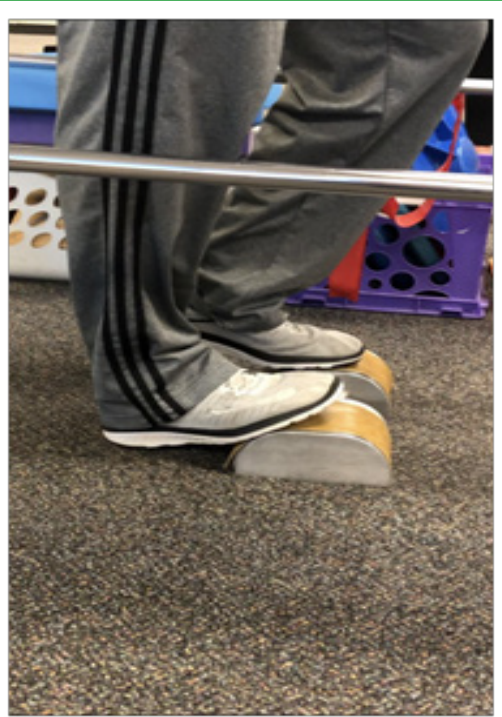

Figure 4.2: Eccentric calf exercise with RLE slowly descending heel for eccentric contraction on RLE. 
Gait training activities were performed in the parallel bars for stability and an improvement in independent walking. Gait training activities incorporated forward and backward walking without upper extremity employment for initiation of heel-toe sequence. Further, sidestepping in the parallel bars was utilized to heighten stability and functional strength in the gluteus medius muscle. Other activities such as static squatting in parallel bars with a half foam roller, under gait activities, included enhancing the strength of anterior tibialis muscle for an improvement in dorsiflexion for heel striking within the gait cycle. This activity influences an increase in quadriceps strength from the static squatting along with the initiation of the anterior tibialis muscle from the positioning of the feet on the foam roller. Assistant device training was utilized with a rolling walker for household and some community ambulation. The patient was taught how to use appropriate weight on the rolling walker from upper extremity for the advancement of right lower extremity primarily. This portion of education was useful for the deterrence of overly compensating upper extremities while walking for potentially risking overuse and or carpal tunnel in the future. Then, the patient progressed from RWW to forearm crutches for household and community ambulation. Also, during gait and weight-bearing, the subject showed no obvious right knee hyperextension, and minimal to no toe drag while ambulating. Lastly, a recumbent stepper like the Nustep was utilized in gait activities for gain in endurance and activation of hip flexion. The patient performed this activity with intensity on level 6 and was set to five to six minutes, with intervals of 30 seconds fast and 10 seconds medium speed.

Each exercise progressed when activities became too comfortable and non-beneficial. Activities highly difficult or unable to perform were withdrawn from the plan of care. The subject was in the outpatient neurology clinic at Baylor Scott and White in Grapevine, Texas, for approximately one year. At first, the patient was being seen three times a week for two months. Due to insurance, the patient's frequency and duration decreased to one to two times a week for two months at a time. There were some improvements based on functional scores seen in Table 4, comparing initial testing from the last revaluation. Even though the patient did reach all PT set goals or personal goals, there was a dramatic increment in functionality, with the patient displaying the possibility of improvement. The client will continue with skilled physical therapy along with the continuation of his home exercise program for an increase in improvement in the deficits listed above and increase functional mobility for independence.

\begin{tabular}{|l|l|l|l|l|}
\hline \multicolumn{2}{|c|}{ Test and Measures } & \multicolumn{2}{c|}{ Initial Evaluation } & \multicolumn{2}{c|}{ Re-evaluation } \\
\hline MMT & Left & Right & Left & Right \\
\hline Hip Flexion & $3 / 5$ & $+1 / 5$ & $+4 / 5$ & $+2 / 5$ \\
\hline Knee Extension & $4 / 5$ & $-2 / 5$ & $5 / 5$ & $+3 / 5$ \\
\hline Knee Flexion & $4 / 5$ & $-2 / 5$ & $5 / 5$ & $+3 / 5$ \\
\hline Ankle Dorsiflexion & $4 / 5$ & $-1 / 5$ & $5 / 5$ & $2 / 5$ \\
\hline Ankle Plantar Flexion & $-3 / 5$ & $-2 / 5$ & $3 / 5$ & $+1 / 5$ \\
\hline FTSTS & 26.17 second & 19.59 second \\
\hline TUG & 45.74 second w/ RWW & 26 seconds w/ forearm crutches \\
\hline 6MWT & 200 feet w/ RWW & 450 feet w/ RWW \\
\hline
\end{tabular}

\section{Discussion}

In this case study, the patient was unable to reach his personal goal of walking independently with no restrictive devices. Withal, he did meet the majority of set physical therapy goals and show progression in functional activities inside and outside the clinic. Due to his diagnosis, the patient's personal goal was not achievable within the time period. With the patients' rare diagnosis, minimal literature was available for the appropriate interventions for these specific symptoms. Therefore, the interventions that were selected and based on the patients' specific impairments. These sequences of interventions have provided a significant improvement in the overall function of the present patient. Evidence supports using the exercises utilized in this case study for each specific impairments [11-15].

The outcomes related to this case report suggested gastrocnemius weakness as the leading cause for the patients' right knee hyperextension during the midstance phase in the gait cycle. Commonly, quadriceps weakness and gastrocnemius tightness are typically the explanation for the right knee hyperextension. To our amazement, that was not the case for this particular patient. Prior to the previous approach and reasoning, the patient was participating in targeting quadriceps strengthening exercises and gastrocnemius stretching for several months and showed no improvement. After administering gastrocnemius strengthening exercises, the patient showed improvement by reducing knee hyperextension during midstance. There is literature supporting the strong relationship between ankle plantar flexor weakness and knee hyperextension during midstance [15]. Additionally, due to this finding and improvements, the patient was able to progress from RWW to forearm crutches for household and community ambulation in a safe manner. Approximately one year of physical therapy facilitated; increased in strength, gait mobility, motor control, balance, and function. Another explanation the patient responded so satisfactorily with therapy was because of the patients' compliance with his home exercise program and the support and encouragement of his wife and kids.

\section{Conclusion}

The current case report had the purpose of illustrating the rare clinical implications of an individual with Transverse Myelitis. This case study can give an outline of the outpatient rehabilitation process for patients with this diagnosis. Multiple constraints occur along the way, starting with understanding this particular case presentation, and finding adequate literature supporting said case interventions. Comparable to complex medical diagnosis, physical therapists emphasize treating the symptoms for a favorable outcome in combination with the patient's goals. One of the limitations and challenges of this study was that TM is a rare diagnosis with minimal evidence of patients being treated. Based on the patient's functional level, the decisions that were made seemed to be appropriate given the presentation, prognosis, and outcomes that he will continue to achieve throughout his remaining time in treatment. Moreover, our clinical observation for this patients' right knee hyperextension during mid-stance, suggests a possible interventions program focusing on an alternative route, for the improvement in gait mobility for this patient. Future studies attending similar cases should focus 
on examining long term benefits of physical therapy as well as a more extended period of intervention to obtain a clear clinical picture of the advantages of such treatment.

Conflicts of interest/Competing interests: Authors report no conflict or competing interest.

Consent to participate: The participant gave signed consent for this case report.

Authors' contributions: All authors contributed to the study conception and design.

\section{References}

1. Transverse Myelitis Fact Sheet. National Institute of Neurological Disorders and Stroke.

2. Cooper A, Alghamdi GA, Alghamdi MA, Altowaijri A, Richardson S. The Relationship of Lower Limb Muscle Strength and Knee Joint Hyperextension during the Stance Phase of Gait in Hemiparetic Stroke Patients. Physiotherapy Research International. 2011;17(3):150-156. doi:10.1002/pri.528.

3. Transverse Myelitis. NORD (National Organization for Rare Disorders).

4. Khallaf, M.E., Gabr, A.M., \& Fayed, E.E. (2014). Effect of Task Specific Exercises, Gait Training, and Visual Biofeedback on Equinovarus Gait among Individuals with Stroke: Randomized Controlled Study. Neurology Research International. 2014:1-9. doi:10.1155/2014/693048.

5. Five Times Sit to Stand Test. Shirley Ryan AbilityLab.

6. Timed Up and Go. Shirley Ryan AbilityLab. https://www.sralab. org/rehabilitation-measures/timed-and-go\#spinal-injuries. Accessed April 1, 2020

7. Rubin M, By, Rubin M, Last full review/revision Jan 2020 Content last modified Jan 2020. Acute Transverse Myelitis Neurologic Disorders. Merck Manuals Professional Edition.

8. Martinuzzi, A., Salghetti, A., Betto, S, et al. (2010). The International Classification of Functioning Disability and Health, version for children and youth as a roadmap for projecting and programming rehabilitation in a neuropaediatric hospital unit. Journal of Rehabilitation Medicine. 42(1):49-55. doi:10.2340/16501977-0468.

9. Inserm. Orphanet: Idiopathic acute transverse myelitis. Orphanet: Idiopathic acute transverse myelitis.

10. 6 Minute Walk Test. Shirley Ryan AbilityLab. https://www. sralab.org/rehabilitation-measures/6-minute-walk-test. Accessed April 1, 2020.

11. Freitas, G.R.D., Szpoganicz, C., \& Ilha, J. (2018). Does Neuromuscular Electrical Stimulation Therapy Increase Voluntary Muscle Strength After Spinal Cord Injury? A Systematic Review. Topics in Spinal Cord Injury Rehabilitation. 24(1):6-17. doi:10.1310/sci16-00048.

12. What is Transverse Myelitis (TM)?: Johns Hopkins Transverse Myelitis Center. What is Transverse Myelitis (TM)? Johns Hopkins Transverse Myelitis Center. https://www. hopkinsmedicine.org/neurology_neurosurgery/centers_clinics/ transverse_myelitis/about-tm/what-is-transverse-myelitis.html. Published October 5, 2015. Accessed April 1, 2020.

13. Yun, S-M., Park, S-K., \& Lim, H.S. (2017). Influence of pilates training on the quality of life of chronic stroke patients. Journal of Physical Therapy Science. 29(10):1830-1835. doi:10.1589/ jpts.29.1830.

14. O'Sullivan, S.B. (2007). Examination of Motor Function: Motor Control and Motor Learning. In: O'Sullivan SB. Physical Rehabilitation. 5th ed. Philadelphia, PA: F.A. Davis. 227-271.
15. Franchi MV, Reeves ND, Narici MV. Skeletal Muscle Remodeling in Response to Eccentric vs. Concentric Loading: Morphological, Molecular, and Metabolic Adaptations. Frontiers in Physiology. 2017;8. doi:10.3389/fphys.2017.00447. 